\title{
HACKATHONS NO SETOR PÚBLICO BRASILEIRO: OBJETIVOS E RESULTADOS SOB A ÓTICA DE AGENTES PÚBLICOS PROMOTORES DAS INICIATIVAS
}

\author{
HACKATHONS IN BRAZILIAN PUBLIC SECTOR: PURPOSES \\ AND RESULTS FROM THE PERSPECTIVE OF PUBLIC AGENTS \\ PROMOTING THE INITIATIVES
}

\section{HACKATHONS EN EL SECTOR PÚBLICO BRASILEÑO: METAS Y RESULTADOS BAJO LA PERSPECTIVA DE LOS AGENTES PÚBLICOS PROMOTORES DE ESTAS INICIATIVAS}

Gabriel de Deus Ferreira

Mestre em Administração - PPGA/UnB, Brasília, Brasil

Pesquisador - UnB, Brasília, Brasil

gabriel.dfer@gmail.com

\section{Josivania Silva Farias}

Doutora em Administração - PPGA/UnB, Brasília, Brasil

Professora de Graduação e de Pós-Graduação em Administração - UnB, Brasília, Brasil

josivania@unb.br

\author{
Contextus \\ ISSNe 2178-9258 \\ Organização: Comitê Científico Interinstitucional \\ Editor-Chefe: Diego de Queiroz Machado \\ Editora Associada: Alane Siqueira Rocha \\ Avaliação: double blind review pelo SEER/OJS \\ Recebido em 08/11/2018 \\ Aceito em 22/01/2019 \\ $2^{\text {a }}$ versão aceita em $20 / 02 / 2019$ \\ http://dx.doi.org/10.19094/contextus.v17i1.39555
}

\section{RESUMO}

O setor público vem convocando cidadãos com competências em programação (hackers) para maratonas de soluções digitais de interesse coletivo, os hackathons. Esta pesquisa teve como objetivo investigar a finalidade e resultados das iniciativas de hackathons utilizadas como estratégia para inovação aberta no setor público brasileiro, considerando a perspectiva de agentes públicos promotores das iniciativas. Trata-se de estudo qualitativo e exploratório-descritivo. A partir de múltiplos casos, foram realizadas análise documental e oito entrevistas. Aplicou-se análise de conteúdo ao corpus de evidências empíricas. Foram identificados 47 hackathons realizados até 2016, observando-se um aumento de sua ocorrência ao longo dos anos. Essas iniciativas visam: gerar inovações, promover participação social, aumentar a transparência e aproximar os atores entre si. Como fragilidade, tem-se a baixa sustentabilidade das soluções desenvolvidas. Por fim, propõe-se um framework de promoção de hackathons no setor público.

Palavras-chave: hackathon; inovação aberta; setor público; governo; citizen-sourcing.

\begin{abstract}
The public sector has been inviting citizens with programming skills (hackers) for marathons to propose digital solutions of collective interest, the hackathons. This research aimed to investigate the purpose and results of hackathon initiatives used as a strategy for open innovation in the Brazilian public sector, considering the public agents' perspective regarding the initiatives. This is a qualitative, exploratory-descriptive study of multiple cases, based on document analysis and eight interviews. We identified 47 hackathons promoted until 2016 and observed an increase in the occurrence of such events. These initiatives main objectives in public sector are to create innovation, to promote social participation, to strengthen transparency and to bring actors closer to one another. As a weak spot, the low sustainability of the developed solutions was observed. A framework to promote hackathons in the public sector is proposed.
\end{abstract}

Keywords: hackathon; open innovation; public sector; government; citizen-sourcing. 


\section{RESUMEN}

El sector público viene convocando a ciudadanos con competencias en programación (hackers) para maratones de proposición de soluciones digitales de interés colectivo, los hackathons. Este trabajo tuvo como objetivo investigar la finalidad y resultados de las iniciativas de hackathons utilizadas como estrategia para innovación abierta en el sector público brasileño, considerando la perspectiva de agentes públicos promotores de las iniciativas. Se trata de un estudio cualitativo y exploratorio-descriptivo. A partir de múltiples casos, se realizaron análisis documentales y ocho entrevistas. Se aplicó análisis de contenido al corpus de evidencias empíricas. Se identificaron 47 hackathons realizados hasta el 2016, observándose un aumento de su ocurrencia a lo largo de los años. Estas iniciativas sugeren generar innovaciones, promover la participación social, aumentar la transparencia y acercar a los actores los unos a los otros. Como fragilidades, se tiene la sostenibilidad de las soluciones desarrolladas. Por último, se propone un framework de promoción de hackathons en el sector público.

Palabras clave: hackathon; innovación abierta; sector público; gobierno; citizen-sourcing.

\section{INTRODUÇÃO}

Devido às grandes mudanças políticas, sociais e econômicas, os agentes públicos estão cada vez mais pressionados a criar soluções para problemas que enfrentam todos os dias. $\mathrm{O}$ envolvimento do cidadão é uma das formas encontradas para lidar com a escassez de recursos e a dificuldade de inovar (THAPA et al., 2015). O tratamento do cidadão não apenas como usuário, mas como participante ativo na prestação de serviços públicos é elemento chave para a inovação desses serviços (HILGERS; IHL, 2010).

Diversas iniciativas surgem com objetivo de promover uma maior transparência, colaboração e participação dos cidadãos ao construir soluções para problemas da administração pública. É o caso dos hackathons, maratonas que reúnem programadores, desenvolvedores e inventores, os hackers - geralmente motivados pelo reconhecimento, recompensas financeiras e aprendizado - para criar projetos que transformem informações de interesse público em soluções digitais, acessíveis a todos os cidadãos (FERREIRA; SILVA, 2018).

A partir de uma pesquisa documental, realizada em dezembro de 2016 no Diário Oficial da União e em portais de notícias, detalhada na seção de resultados, identificaram-se 47 hackathons já promovidos, sendo o primeiro de 2012. Essas iniciativas aconteceram em diversas regiões do país, assim como em todos os níveis de governo (municipal, estadual e federal).

Tradicionalmente, as organizações do setor público executam as atividades necessárias à melhoria de um serviço, normalmente por servidores públicos. A terceirização (outsourcing) da execução de tarefas ao cidadão (citizen) mostra uma nova relação entre setor público e cidadão (LUKENSMEYER; TORRES, 2008).

Denominada de citizen-sourcing, a chamada aberta aos cidadãos para que interajam com o governo com a finalidade de gerar valor público ou de inovar em soluções vem modificando a forma como são operados os processos administrativos do setor público. Os propósitos do 
citizen-sourcing podem ser: participação social; aumento da transparência; inovação organizacional; melhoria na qualidade tanto das decisões quanto da imagem institucional a partir de conhecimentos e habilidades profissionais específicas; ou geração de ideias inovadoras (OECD, 2001; FREITAS; DACORSO, 2014; NAM, 2012).

Por se tratar de um tema novo na agenda de pesquisa e de agentes públicos, ainda são incipientes as discussões sobre inovação aberta no setor público e o emprego de iniciativas como os hackathons para esse tipo de propósito (FERREIRA et al., 2016). Além disso, ainda se desconfia da capacidade do cidadão em auxiliar na resolução de problemas do setor público (THAPA et al., 2015). Tanto a academia quanto organizações do setor público não delimitam claramente quais os objetivos e resultados esperados com iniciativas de inovação aberta no setor público nem verificam se esses processos estão alcançando a participação esperada (POWELL; COLIN, 2008). Afinal, quais são os objetivos dos hackathons no setor público brasileiro? Estas iniciativas trazem os resultados esperados de uma estratégia de inovação aberta?

Sendo assim, esta pesquisa teve como objetivo geral investigar a finalidade e os resultados das iniciativas de hackathons utilizadas como estratégia para inovação aberta no setor público brasileiro, considerando a perspectiva de agentes públicos promotores das iniciativas. Assim, formulam-se os seguintes objetivos específicos desta pesquisa: a) mapear as iniciativas de hackathons no setor público; b) investigar os objetivos e resultados dos hackathons a partir dos relatos de agentes públicos promotores desse tipo de iniciativa; e c) propor um framework de promoção de hackathons no setor público.

\section{REFERENCIAL TEÓRICO}

Abrindo esta seção teórica, torna-se necessário um breve relato da trajetória da Administração Pública enquanto objeto de estudo, visando compreender como se chegou ao contexto atual, em que a inovação passa a fazer parte das estratégias de governos e demais instituições públicas. Segundo Osborne (2010), para resultar no atual design de produção e entrega de serviços públicos - um modo aberto, negociado com diversos atores, numa perspectiva pluralista -, é preciso entender que houve perspectivas ou mesmo paradigmas anteriores da gestão pública, os quais foram evoluindo até aqui.

A partir do final do século XIX até o fim dos anos 1970 e início dos anos 1980, a implementação de políticas públicas e a entrega de serviços públicos eram baseadas na tradição estatista e burocrática da Public Administration (PA), cuja natureza do estado era unitária, o 
foco se dava no sistema político, a natureza do sistema de serviços era fechada e o valor básico estava vinculado ao ethos do setor público (OSBORNE, 2010).

Dos anos 1990 até o início do século XXI, surge uma segunda abordagem denominada New Public Management (NPM), em que a natureza do estado passa a ser regulatória, o foco centra-se na organização, com ênfase em gestão de recursos organizacionais e performance. Inicia-se um ritual de abertura em termos de natureza do sistema de serviços, deslocando-se os valores básicos para aqueles valorizados pelo mercado, como eficácia, competição e marketplace (OSBORNE, 2010).

Por fim, do início do século XXI até os dias atuais, mais uma vez a perspectiva (alguns até exageram ou arriscam denominar de 'paradigma') da administração pública se desloca mais uma vez para uma New Public Governance (NPG), cuja natureza do estado passa a ser plural/pluralística, o foco contextual está na organização, mas também em seu ambiente/entorno, passa-se a valorizar relações, negociação de valores, a natureza do sistema de serviços passa a ser aberto-fechado (open closed) e a base de valores passa a ser dispersa e contestada (OSBORNE, 2010). Chega-se à fase das networks, das relações, da pluralidade de atores que passam a protagonizar, também, sobre assuntos de governo. Um desses atores, no qual este trabalho se concentra, é o cidadão, que por meio de estratégias de citizensourcing tem colaborado com a gestão pública e suas mudanças.

Isso enseja, perfeitamente, a compreensão do conceito de inovação aberta e seu advento no setor público. Para Chesbrough et al. (2006, p. 1), a inovação aberta é o "uso proposital de conhecimentos internos e externos à organização para acelerar inovação interna, e expandir mercados $[\ldots]$ ". Enquanto as inovações no setor privado normalmente objetivam a vantagem competitiva e adição de valor em termos de receitas, no setor público a inovação tem como objetivo principal a melhoria da prestação de serviços ao cidadão (BOMMERT, 2010).

O setor público está sujeito a regulações que determinam sua interação com a sociedade. O governo geralmente não está apto a formular a entes externos questões sobre intervenções voltadas à resolução de problemas, à reinvenção de serviços públicos ou mesmo ao aumento da transparência (MERGEL; DESOUZA, 2013).

A inovação aberta no setor público pode ser definida a partir de quatro fases (MERGEL, 2015). No modelo da autora, a pré-fase tem como objetivo a definição de um problema de gestão pública, ou seja, aquele problema que o governo gostaria de contar com a ajuda dos cidadãos para solucioná-lo. A primeira fase é de geração de ideias, com a promoção e distribuição de requisitos dos concursos, onde cidadãos amadores e profissionais submetem ideias para resolução do problema público. As ideias são coletadas, dando início à fase de 
incubação, quando os cidadãos votam pelos projetos preferidos e colaboram enviando ideias. Na terceira fase, o gestor da inovação aberta pede sugestões sobre implementação, com a revisão e seleção das ideias, principalmente pelos critérios de legalidade, sustentabilidade e viabilidade. A última fase é a de resultados e implementação - momento em que ocorre o refinamento, a implementação e a institucionalização da prática. Observa-se por essas demarcações que o cidadão exerce papel de conselheiro, auxiliando na concepção e definições, e não em sua implementação de fato.

Citizen-sourcing é a relação entre cidadão e governo, auxiliando-o na busca por mais responsividade e efetividade (LINDERS, 2012). O governo é o principal responsável pela interação, mas os cidadãos influenciam a direção e o resultado, inclusive na criação, execução e monitoramento de serviços públicos. Para o autor, essa interação se dá em fases. Na primeira fase (design), os cidadãos contribuem com ideias e sugestões para os serviços e programas do governo. As outras fases são a de execução (entrega de soluções e operações do dia a dia) e a de monitoramento (quando o cidadão avalia ou monitora serviços públicos e a operação das organizações públicas).

O cidadão deixa de ser apenas consumidor de um serviço público para ser um ator com papel ativo na construção de uma solução para um problema. O citizen-sourcing não pressupõe ambiente on-line e uma multidão de cidadãos, apenas o envolvimento do cidadão como voluntário em uma tarefa de objetivo público (NAM, 2012). O resultado da contribuição do cidadão (com recursos, tempo, conhecimento e esforço) para alcançar um objetivo comum, sugere responsabilidade compartilhada entre organizações do setor público e cidadão (citizen) na solução de problemas (LINDERS, 2012).

As estratégias de citizen-sourcing podem trazer benefícios como: melhoria da consciência de problemas sociais, implementação de práticas mais efetivas na experiência do cidadão, aumento da confiança entre governo e cidadãos; melhoria da imagem institucional; solução de problemas; e maior transparência nas informações públicas (REDDEL; WOOLCOCK, 2004; POWELL; COLIN, 2008; GAVENTA; BARRETT, 2010; HILGERS; IHL, 2010; NAM, 2012). Além disso, o contato com vários atores internos e externos à organização pode potencializar a inteligência coletiva, possibilitada pela complementaridade de informações e conhecimentos da interação desses atores (BRABHAM, 2013).

De acordo com a Organização para a Cooperação e Desenvolvimento Econômico (OECD, 2001), a participação de cidadãos na construção de políticas é investimento para a boa governança pública. Permite ao governo ter acesso a informações, perspectivas e potenciais 
soluções, aumentando a qualidade das decisões e a confiança no governo, bem como fortalecendo a democracia e a capacidade cívica.

Estudos recentes buscam entender o papel dos Laboratórios de Inovação, Laboratórios Hackers e Living Labs no processo de inovação aberta no setor público. Para Gascó (2016), os Living Labs são espaços em que cidadãos e outras organizações podem se encontrar para experimentar novas soluções, políticas e ferramentas de governança. Para Yazdizadeh e Tavasoli (2016), os laboratórios também têm como propósito a colaboração para resolução de problemas. São considerados intermediários, com objetivo de mediar a relação entre usuários e organizações do setor público e privado para capturar insights de usuários de serviços públicos. Esses laboratórios atuam no suporte às empresas para criar um ecossistema de inovação que beneficie tanto o setor público, quanto o privado, enfatizando a participação dos cidadãos. Podem-se observar casos de laboratórios em algumas organizações do governo brasileiro, como o Laboratório Hacker da Câmara dos Deputados, o MobiLab da Secretaria de Transporte da Prefeitura de São Paulo e o DataPOA da Prefeitura de Porto Alegre.

Entretanto, algumas críticas são levantadas a respeito das iniciativas de citizen-sourcing e inovação aberta no setor público. Nam (2012) ressalta que os objetivos da inovação aberta no setor público podem ser determinados por uma real necessidade de melhoria na prestação de serviços públicos, ou apenas para "parecer legal” ( $\mathrm{cool}$ ) ou modismo.

Quando o objetivo é parecer "cool", um dos propósitos pode ser o de melhoria da imagem institucional. Porém, quando a perspectiva é orientada às realizações que são necessárias (perspectiva “hot”), então é possível: criar informações com os cidadãos, melhorar serviços com os cidadãos, desenvolver soluções com os cidadãos ou mesmo formular políticas públicas com os cidadãos (NAM, 2012).

O concurso é uma das estratégias de citizen-sourcing mais utilizadas pelos governos (NAM, 2012). Conforme afirmam Mergel e DeSouza (2013), apesar de os concursos ainda possuírem barreiras institucionais para serem implementadas (como a burocracia e dificuldades para distribuição de recompensas financeiras), passam a ser uma estratégia mais simples para resolver problemas da administração pública. A justificativa é que os processos de busca por inovações no setor público são muito burocráticos, envolvem especificações detalhadas, pedidos de propostas, licitações, seleções e difíceis negociações contratuais. Sendo assim, concursos que abarquem um maior envolvimento do cidadão na resolução de um problema, como os hackathons, podem ser importantes para possibilitar um maior nível de inovação aberta no setor público. 
O termo hackathon surgiu em 1999 em uma iniciativa de um grupo de desenvolvedores de softwares do sistema operacional OpenBSD em Calgary, Canadá. O evento aconteceu com o objetivo de evitar problemas legais relacionados à exportação de criptografia de software e regulações dos Estados Unidos (BRISCOE; MULLIGAN, 2014).

Atualmente, esse tipo de iniciativa já é disseminado globalmente e vem sendo utilizado principalmente quando profissionais com alto nível de competência em desenvolvimento de softwares e design interagem entre si para colaborar com o desenvolvimento de novas soluções - frequentemente software ou aplicação web - patrocinado por alguma empresa ou organização pública. Geralmente, os hackers são atraídos por recompensas pelas melhores soluções desenvolvidas, reconhecimento perante a comunidade hacker, aprendizado ou por pura diversão (FERREIRA; SILVA, 2018).

Ao redor do mundo experiências de hackathons demonstraram o potencial de geração de soluções inovadoras, principalmente no setor de saúde (ANGELIDIS et al., 2016; HYNES et al., 2016). Angelidis et al. (2016) apresentam casos desses eventos na Colômbia, Uganda, Grécia e México, onde profissionais ligados às Tecnologias da Informação e Comunicação, interessados em promover o impacto social, desenvolvem tecnologias em saúde baseadas em celulares, principalmente para populações de baixa ou média renda. De acordo com os autores, os hackathons promovem o encontro de pessoas com diversas experiências para resolver problemas em competições de curta duração nas quais as equipes desenvolvem soluções inovadoras. Além do recente interesse nas iniciativas de hackathons no setor público, percebese quão pouco a literatura aborda suas possíveis consequências negativas. No setor público, espera-se que, durante o concurso, sejam criados "projetos que transformem informações de interesse público em soluções digitais, acessíveis a todos os cidadãos" (CÂMARA DOS DEPUTADOS, 2014).

Estudos levando em consideração a percepção do agente público, em vários países, contextos e períodos, normalmente citam os mesmos pontos sobre o envolvimento de cidadãos em projetos de inovação: a) cidadãos não possuem as competências necessárias; b) são desmotivados e não estão dispostos a contribuir; c) não compreendem as especificidades da administração pública; d) possuem interesses pessoais e agenda própria (YANG, 2005; KARLSSON et al., 2012; THAPA et al., 2015).

O estudo de Thapa et al. (2015), baseado em um questionário com 358 agentes públicos municipais da Alemanha, revela que, apesar da crescente pressão por inovação percebida pelos agentes públicos, $70 \%$ dos respondentes disseram possuir equipe reduzida para implementação da inovação e $28 \%$ acreditam não possuir know-how dentro da organização para conduzir a 
construção da inovação. Em contrapartida, apenas 13\% dos respondentes consideraram os cidadãos como importantes atores na implementação dessas inovações. É possível perceber que o agente público possui um papel importante na promoção de iniciativas de inovação aberta, sendo necessária a discussão de suas percepções sobre como iniciativas de citizen-sourcing podem promover a inovação aberta no setor público.

Em seguida, é apresentado o Quadro 1 com os principais objetivos e benefícios da promoção de hackathons no setor público de acordo com as referências teóricas apresentadas nesta seção. Esses objetivos e benefícios servirão para a análise de conteúdo dos resultados apresentados na seção Resultados.

\begin{tabular}{|c|c|c|}
\hline $\begin{array}{l}\text { Categorias de objetivos } \\
\text { e benefícios encontradas } \\
\text { na pesquisa }\end{array}$ & Descrição & Referências \\
\hline Aumento da transparência & $\begin{array}{l}\text { Transformar informações de interesse público } \\
\text { acessível a todos os cidadãos. Busca por maior } \\
\text { transparência nas informações públicas, abertura } \\
\text { de dados, geração de informação e Accountability. }\end{array}$ & $\begin{array}{l}\text { Gascó (2016), Hilgers e Ihl } \\
\text { (2010), Freitas e Dacorso } \\
\text { (2014) e Nam (2012) }\end{array}$ \\
\hline Geração de inovações & $\begin{array}{l}\text { Envolve a ideação e construção de soluções para } \\
\text { resolução de problemas. Implementação de } \\
\text { práticas ou ideias mais eficientes e efetivas em } \\
\text { serviços públicos. }\end{array}$ & $\begin{array}{l}\text { Gascó (2016), Lukensmeyer } \\
\text { e Torres (2008), Gaventa e } \\
\text { Barrett (2010), Nam (2012), } \\
\text { Mergel e Desouza (2013), } \\
\text { Bommert (2010) e Brabham } \\
\text { (2013) }\end{array}$ \\
\hline $\begin{array}{l}\text { Melhoria da imagem } \\
\text { institucional }\end{array}$ & $\begin{array}{l}\text { Aumento da confiança entre governo e cidadãos, } \\
\text { parecer legal (cool) e fortalecimento da confiança } \\
\text { no governo. }\end{array}$ & $\begin{array}{l}\text { Reddel e Woolcock (2004), } \\
\text { Nam (2012), OECD (2001) e } \\
\text { Lukensmeyer e Torres } \\
\text { (2008) }\end{array}$ \\
\hline Redução de custos & $\begin{array}{l}\text { Oportunidades para redução de custos para o setor } \\
\text { público. }\end{array}$ & Nam (2012) \\
\hline Participação social & $\begin{array}{l}\text { Envolve aspectos de participação dos cidadãos, } \\
\text { criação de um ambiente de alta valorização da } \\
\text { colaboração com cidadão, coprodução de serviços } \\
\text { e fortalecimento da democracia e capacidade } \\
\text { cívica. }\end{array}$ & $\begin{array}{l}\text { Nam (2012), OECD (2001), } \\
\text { Brabham (2013) e } \\
\text { Lukensmeyer e Torres } \\
\text { (2008) }\end{array}$ \\
\hline Aprendizagem & $\begin{array}{l}\text { Troca de conhecimento e aprendizado em rede e } \\
\text { aproveitamento do valor da inteligência coletiva. }\end{array}$ & $\begin{array}{l}\text { Chesbrough (2003) e Nam } \\
(2012)\end{array}$ \\
\hline $\begin{array}{l}\text { Aceleração de mudanças } \\
\text { organizacionais }\end{array}$ & $\begin{array}{l}\text { Preparação do corpo estatal para o processo de } \\
\text { inovação aberta. }\end{array}$ & Freitas e Dacorso (2014) \\
\hline $\begin{array}{l}\text { Melhoria da qualidade das } \\
\text { decisões }\end{array}$ & Aumento da qualidade das decisões. & OECD (2001) \\
\hline $\begin{array}{l}\text { Melhoria da consciência } \\
\text { de problemas sociais }\end{array}$ & $\begin{array}{l}\text { Conhecimento da população de problemas de } \\
\text { outros cidadãos. }\end{array}$ & Powell e Collin (2008) \\
\hline Formulação de políticas & $\begin{array}{l}\text { Apoiar na criação de novas políticas públicas para } \\
\text { os cidadãos. }\end{array}$ & Nam (2012) e OECD (2001) \\
\hline $\begin{array}{l}\text { Evitar problemas legais } \\
\text { relacionados à criação de } \\
\text { softwares }\end{array}$ & $\begin{array}{l}\text { Evitar problemas relacionados a propriedade } \\
\text { intelectual de soluções digitais. }\end{array}$ & Briscoe e Mulligan (2014) \\
\hline
\end{tabular}

Fonte: elaboração própria. 
As experiências de citizen-sourcing e hackathons apresentadas mostram que é possível desenvolver soluções para problemas no governo a partir da participação social em um curto espaço de tempo (durante uma maratona, por exemplo). Com isso, o citizen-sourcing pode ser uma estratégia importante para auxiliar os agentes públicos na implementação de inovações.

\section{METODOLOGIA}

Este estudo é de natureza exploratório-descritiva. Exploratória por tratar de um fenômeno relativamente recente na realidade brasileira, que é o caso da promoção, pelo setor público, de esforços de engajamento social para implementar soluções de problemas públicos por meio de eventos hackathons, como uma modalidade do esforço de citizen-sourcing. Descritiva porque descreve os propósitos das organizações do setor público na promoção das iniciativas, torna a tarefa empírica também um trabalho descritivo (GODOY, 1995).

Quanto ao método, é possível classificar a pesquisa como um estudo de múltiplos casos (ALVES-MAZZOTTI, 2006). O trabalho tem abordagem qualitativa e a coleta de dados ocorreu por meio de pesquisa documental e entrevistas semiestruturadas. O recorte temporal é transversal, pois os dados foram coletados em um único período de tempo (CRESWELL, 2014).

Para o primeiro objetivo de mapear as iniciativas de hackathon promovidas pelo setor público brasileiro foi utilizado o procedimento de pesquisa documental (BARDIN, 1977). A coleta das evidências documentais se deu em websites, editais de convocação e notícias geradas a partir das iniciativas de hackathons, sem delimitação temporal, nível de governo (municipal, estadual e federal, ou até entre legislativo, executivo e judiciário) ou local de realização, a fim de ampliar a abrangência do mapeamento. Foram conduzidas buscas no dia 01.12.2016 desses documentos no Diário Oficial da União (canal oficial de convocação do setor público - portal. imprensanacional.gov.br/), Google News (website de busca de notícias - news. google.com.br/) e o website Ping Hacker (portal de notícias e divulgação de eventos especializado no público hacker brasileiro - pinghacker.com.br/). Como parâmetros de busca foram utilizadas as palavras-chave "hackathon" e "maratona hacker" com combinação da palavra-chave referente ao locus da pesquisa: "governo" ou "setor público". Para os websites Ping Hacker e Imprensa Nacional (Diário Oficial da União), foram analisados todos os resultados e catalogados aqueles que diziam respeito aos hackathons organizados pelo setor público, excluindo os resultados repetidos ou não condizentes com o objetivo da pesquisa. Já para o website Google News, devido à quantidade de retornos da busca, foram analisados os resultados até a página 15 do 
website, estando o último evento identificado na página 10. Não foram considerados os eventos organizados por entidades privadas sem fins lucrativos (ex.: SEBRAE, Transparência Brasil, entre outros).

A partir dessas buscas, foram catalogados 47 hackathons organizados pelo governo. $\mathrm{Na}$ análise documental (BARDIN, 1977), classificaram-se os hackathons de acordo com as seguintes categorias: local de realização, data de ocorrência, organização realizadora, nível de atuação (municipal, estadual e federal), duração do evento (em dias) e se existia premiação financeira aos vencedores.

Considerando o segundo objetivo, para investigar os objetivos e resultados dos hackathons, foram conduzidas oito entrevistas semiestruturadas com agentes públicos promotores desse tipo de iniciativa no Brasil. A escolha dos sujeitos para realização das entrevistas foi de acordo com a intensidade. De acordo com Patton (1990), a lógica de escolha de sujeitos pela intensidade passa pela seleção dos casos com maior experiência (escolha pelo propósito da pesquisa). Esses casos possibilitam aprender com maior profundidade e conseguir informações mais ricas pertinentes ao estudo. Marshall (1996) e Coyne (1997) afirmam que selecionar os casos aparentemente mais produtivos para atingir os objetivos da pesquisa é a forma mais utilizada em pesquisas qualitativas.

Em busca preliminar realizada em outubro de 2016 seguindo o mesmo protocolo de busca mencionado anteriormente, foram identificadas as organizações que haviam promovido hackathons. Foram contatadas as organizações com o maior número de hackathons promovidos, e convidados para a entrevista os sujeitos que participaram da organização das iniciativas. Foram entrevistados nove sujeitos, em um total de oito entrevistas (uma das entrevistas foi realizada com dois participantes, a pedido da organização responsável, para maior riqueza de informações). As organizações onde os dados foram coletados promoveram 20 hackathons, cobrindo mais de $42 \%$ das iniciativas totais catalogadas.

As entrevistas foram realizadas presencialmente ou por intermédio de software de teleconferência (Skype) entre os meses de outubro e novembro de 2016, tiveram duração total de mais de quatro horas e o tempo médio por entrevista foi de 31 minutos. $\mathrm{O}$ áudio das entrevistas foi gravado e em seguida transcrito para a análise de conteúdo ser aplicada.

Foi solicitado ao órgão locus que participassem da entrevista os agentes públicos responsáveis pela realização dos hackathons. Nesse sentido, sujeitos de diferentes níveis hierárquicos foram entrevistados, a saber: Diretor de Ciência, Tecnologia e Inovação; Pesquisador Tecnologista em Informações e Avaliações; Diretor de Comunicação Externa; Coordenadores e Gestores de área; e Secretários Municipais. 
Para investigar os objetivos e resultados dos hackathons, foram realizadas oito entrevistas com agentes públicos promotores desse tipo de iniciativa. Para a análise dos dados, considerou-se importante avaliar a ocorrência das categorias de análise e não sua frequência de aparição, pois "o acontecimento, o acidente e a raridade possuem, por vezes, um sentido muito forte que não deve ser abafado" (BARDIN, 1977, p. 116).

Para a análise de conteúdo aplicada ao corpus da transcrição das entrevistas, foram levantadas categorias a priori de acordo com a literatura exposta na Seção Referencial Teórico e consolidadas no Quadro 1. A emersão de categorias a posteriori também foi considerada na fase de leitura e releitura dos materiais coletados com vistas à categorização de dimensões de análise. Para conferir ao trabalho qualitativo traços mais significativos de relevância, confiabilidade, consistência e qualidade, tanto na elaboração das categorias de análise, quanto na categorização do conteúdo a partir do corpus das entrevistas, foi realizada triangulação de pesquisadores (GUION; DIELH; MCDONALD, 2002; FARMER et al., 2006).

Para a realização desta pesquisa foram seguidos os procedimentos exigidos nas Resoluções Éticas Brasileiras, em especial a Resolução do Conselho Nacional de Saúde (CNS) 510/2016 e do Comitê de Ética em Pesquisa do Instituto de Ciências Humanas da Universidade de Brasília. Esta pesquisa foi aprovada por esse Comitê no dia 04 de setembro de 2016 (Certificado de Apresentação para Apreciação Ética número 57753316.0. 0000.5540).

\section{ANÁLISE E DISCUSSÃO DOS RESULTADOS}

A partir dos procedimentos de busca descritos na Seção Procedimentos Metodológicos, foram catalogados 47 hackathons promovidos pelo setor público brasileiro. A primeira iniciativa foi organizada pela Câmara Municipal de São Paulo, em maio de 2012. Observou-se um crescimento no número de casos ao longo dos anos de 2012 a 2016. No último ano, foram promovidas 17 iniciativas até novembro de 2016, período em que a busca documental foi finalizada. Isso corrobora os achados de Mergel e Desouza (2013) de que estratégias de citizensourcing, como os hackathons, vêm aumentando no setor público. Esse fenômeno também se dá no contexto brasileiro. 
Gráfico 1 - Número de hackathons promovidos pelo setor público por ano.

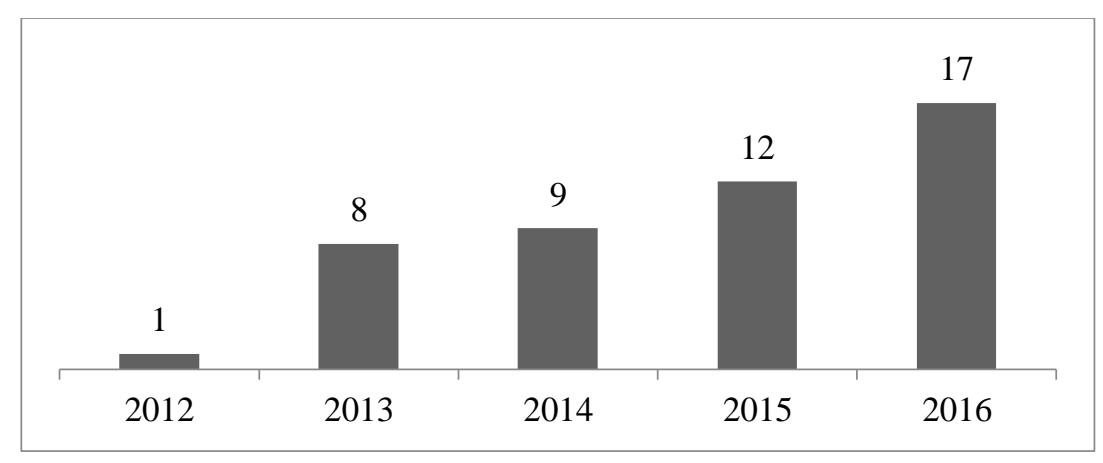

Fonte: elaboração própria.

Organizações públicas do nível Municipal são as que mais organizam hackathons, com 28 iniciativas. Organizações do nível Federal promoveram 11 iniciativas, e do nível Estadual, oito. A maioria das iniciativas se concentrou nos estados de São Paulo, Paraná, Rio Grande do Sul, Pernambuco e no Distrito Federal, responsáveis por $80 \%$ dos casos identificados. Foram identificadas 30 organizações promotoras de hackathons. É possível notar que mais da metade das organizações $(55 \%)$ optou por promover mais de um evento. Destaca-se a Prefeitura da Cidade do Recife, com quatro hackathons promovidos.

As iniciativas apresentam características semelhantes quanto ao formato. São realizadas palestras de abertura e formadas as equipes que irão participar da maratona de desenvolvimento das soluções. As equipes são avaliadas ao final da maratona e premiadas com recompensas financeiras (prêmios de até R\$ 30,000.00) ou não financeiras (viagens, “miniestágios”, cursos, programas de mentoria e incubação, entre outros). A maioria dos hackathons (73\%) dura entre 2 e 3 dias, ocorrendo normalmente aos finais de semana. Esse formato se assemelha às fases da inovação aberta no setor público, destacadas por Mergel (2015). Nesse sentido, é possível observar que os hackathons são promovidos principalmente nas fases iniciais da inovação, isto é, na geração de ideias.

Para alcance do objetivo de investigar os objetivos e resultados dos hackathons, as entrevistas foram analisadas de forma a identificar a presença, ou ocorrência, das categorias definidas a priori. Ressalta-se a emersão de categorias a posteriori que foram incorporadas na análise, a saber: a) fortalecimento do ambiente/rede de inovação; e b) aproximação de atores (setor privado, setor público e instituições de ensino, pesquisa e desenvolvimento). A Tabela 1 descreve a ocorrência (representada com o algarismo 1) ou não ocorrência (algarismo 0) das categorias de análise. As oito entrevistas realizadas estão dispostas em colunas e codificadas como E1 até E8. 
Tabela 1 - Resultado da análise das entrevistas, por categorias e subcategorias de análise

\begin{tabular}{|c|c|c|c|c|c|c|c|c|c|c|}
\hline Objetivos e benefícios da promoção de hackathons & E1 & E2 & $\mathbf{E 3}$ & E4 & E5 & E6 & E7 & $\mathbf{E 8}$ & $\mathbf{n}$ & $\%$ \\
\hline Geração de inovações & 1 & 1 & 1 & 1 & 1 & 1 & 1 & 1 & 8 & 100 \\
\hline Participação social & 1 & 1 & 1 & 1 & 1 & 1 & 1 & 1 & 8 & 100 \\
\hline Aumento da transparência & 0 & 1 & 1 & 1 & 1 & 1 & 1 & 0 & 6 & 75 \\
\hline $\begin{array}{l}\text { Fortalecimento do ambiente/rede de inovação ( } a \\
\text { posteriori) }\end{array}$ & 1 & 1 & 0 & 1 & 1 & 1 & 1 & 0 & 6 & 75 \\
\hline Aceleração de mudanças organizacionais & 1 & 0 & 1 & 1 & 0 & 1 & 1 & 0 & 5 & 63 \\
\hline $\begin{array}{l}\text { Aproximação de atores (setor privado, setor público e } \\
\text { instituições de ensino, pesquisa e desenvolvimento) } \\
\text { para inovação (a posteriori) }\end{array}$ & 1 & 0 & 0 & 1 & 1 & 1 & 1 & 0 & 5 & 63 \\
\hline Aprendizagem & 0 & 1 & 0 & 0 & 1 & 1 & 1 & 0 & 4 & 50 \\
\hline Melhoria da qualidade das decisões & 0 & 1 & 0 & 1 & 0 & 1 & 1 & 0 & 4 & 50 \\
\hline Melhoria da imagem institucional & 0 & 1 & 0 & 1 & 0 & 1 & 0 & 0 & 3 & 38 \\
\hline Melhoria da consciência de problemas sociais & 0 & 1 & 0 & 0 & 0 & 0 & 1 & 1 & 3 & 38 \\
\hline Redução de custos & 1 & 0 & 0 & 0 & 0 & 1 & 0 & 0 & 2 & 25 \\
\hline Formulação de políticas & 0 & 0 & 0 & 0 & 0 & 1 & 0 & 0 & 1 & 13 \\
\hline $\begin{array}{l}\text { Evitar problemas legais relacionados à criação de } \\
\text { softwares }\end{array}$ & 0 & 0 & 0 & 0 & 0 & 0 & 0 & 0 & 0 & 0 \\
\hline
\end{tabular}

Fonte: elaboração própria.

Conforme se pode notar na Tabela 1, os hackathons possuem foco claro de convite aos cidadãos a participarem da resolução de problemas e construção de soluções que beneficiem a sociedade. A geração de inovações e a participação social foram objetivos citados em todas as entrevistas realizadas.

A transparência e abertura de dados no setor público é objeto de muitos hackathons, mencionado em seis entrevistas (75\%). O setor público busca, por meio dos hackathons, o apoio do cidadão para criar soluções que facilitem a abertura, apropriação e visualização de dados. Nesse sentido, os resultados reforçam que o setor público vem adotando iniciativas com objetivo de melhoria da prestação de serviços públicos, construindo essas soluções conjuntamente com os cidadãos, principalmente em relação à transparência e dados abertos, conforme mencionado por Hulgard e Ferrarini (2010).

As organizações do setor público podem ter ações diretas relacionadas à inovação aberta, atuando de duas formas: a) fortalecendo o ambiente ou rede de inovação; e b) aproximando atores importantes para a inovação, como cidadãos, pesquisadores, setor público e setor privado. O uso proposital de fluxos de conhecimento interno e externo acelerara a inovação (CHESBROUGH et al., 2006). Nesse sentido, as estratégias de citizen-sourcing, como os hackathons, atuam não só no fluxo de conhecimento do cidadão para o setor público, mas também entre os cidadãos, organizações públicas e outros atores, fomentando a inovação aberta no setor público e sociedade.

A gente não quer soluções para a prefeitura utilizar. Que as pessoas desenvolvam soluções que sejam usadas pelos próprios cidadãos. E que resolvam os problemas da cidade, que gerem negócios, que as 
pessoas empreendam, que usem os dados abertos. (Entrevista 5)

A promoção de hackathons também fomenta o aprendizado. Em alguns casos, o hackathon é utilizado como forma de aproximar estudantes de ensino superior da prática, principalmente em questões de desenvolvimento tecnológico. Para Nam (2012), as estratégias de citizen-sourcing possibilitam aproveitar o valor da inteligência coletiva. $O$ valor da troca de conhecimentos com atores dentro e fora da organização a fim de gerar novas soluções é aspecto básico da inovação, ressaltado por Chesbrough et al. (2006). O aprendizado pode também ser para membros do próprio setor público, fortalecendo as organizações com novos conhecimentos e capacidades internas.

Engajar cidadãos no processo de planejamento de uma política ou ação é uma "relação avançada de duas vias" entre setor público e cidadão no princípio de parceria (OECD, 2001, p. 16). A participação do cidadão pode melhorar a qualidade da tomada de decisão e aumentar a confiança do cidadão nas organizações do setor público, possibilitando melhores políticas públicas e sua legitimidade. A partir das entrevistas, pôde-se perceber que a participação do cidadão possibilita melhorias na qualidade das decisões dos agentes públicos. Essa melhoria pode se dar tanto pelo próprio contato entre organizações públicas e o cidadão, entendendo melhor suas necessidades, anseios e problemas, como também a partir das soluções desenvolvidas durante os hackathons.

Apesar de a origem dos eventos de hackathons, segundo a literatura, ter sido objetivada pela prevenção de problemas legais em empresas de desenvolvimento de software (BRISCOE; MULLIGAN, 2014), este propósito não foi mencionado pelos agentes públicos entrevistados. Quando questionados sobre o porquê da escolha dos hackathons como estratégia para alcance dos objetivos propostos, foram mencionados três motivos: a) formato da iniciativa traz agilidade na criação de soluções; b) sugestão de um parceiro; e c) modismo - promoveram porque outras organizações estavam fazendo o mesmo.

[...] é um tipo de evento que já esteve mais na moda. E quando a gente começou era o que era mais utilizado pela comunidade hacker. (Entrevista 7)

Esses aspectos reforçam as críticas levantadas a respeito das iniciativas de citizensourcing e inovação aberta no setor público. Conforme ressalta Nam (2012), os objetivos da inovação aberta no setor público podem ser determinados por uma real necessidade de melhoria na prestação de serviços públicos, ou apenas para se parecer legal (cool) - ou modismo. Sendo assim, a partir dos relatos, é possível perceber que, apesar de a origem da realização dos 
hackathons, em alguns casos, ter sido por um modismo, este sempre é acompanhado por outros objetivos, como aumento da transparência e geração de inovações.

O hackathon tem sido promovido principalmente na fase de design (planejamento e ideação de soluções), apesar de possuir aspectos de execução (uma fase de entrega de soluções para o setor público e cidadãos), conforme taxonomia de Linders (2012). Alguns entrevistados destacam que a execução é secundária ao objetivo da iniciativa:

Não, vocês [participantes] não precisam programar. Vocês precisam sentar e pensar em boas ideias, claro que se vocês quiserem fazer um protótipo [...] Mas o importante era na área de produzir boas ideias. (Entrevista 2)

Objetivos mais complexos, que exigem maior aproximação e execução tanto do setor público, como dos cidadãos, foram pouco mencionados pelos agentes públicos, como: redução de custos e formulação de políticas. Com isso, é importante também avaliar os resultados trazidos por essas iniciativas.

Os agentes públicos promotores de hackathon foram questionados a respeito dos resultados e o alcance dos objetivos das iniciativas já realizadas. Em cinco das oito entrevistas, os objetivos foram considerados alcançados. Nesse caso, três aspectos foram destacados: a) aumento do número de novos negócios na região; b) maior apropriação e abertura dos dados por parte da organização pública; e c) mudanças na percepção de funcionários da organização.

Considerando a percepção daqueles que declararam os resultados como parcialmente alcançados, os principais motivos se referiam à qualidade e perenidade das soluções desenvolvidas. Grande parte das soluções é abandonada após a realização do hackathon.

"Existia" aproximadamente 60 aplicativos [...] Houve um Darwinismo do mercado? Houve. Não são todos que estão agora, tem uma meio dúzia deles só que de fato são usados. (Entrevista 6)

A sustentabilidade das soluções é crucial aos esforços de citizen-sourcing. O convite aos cidadãos para participar da construção de soluções, seguido da não implementação dessas ideias, pode gerar descontentamento e até gerar efeito oposto ao esperado, de melhoria da imagem do governo. Outros estudos sobre hackathons, como de Angelidis et al. (2016) e Hynes et al. (2016), reforçam as soluções e envolvimento dos participantes no evento, mas não os resultados e perenidade das soluções desenvolvidas.

Algumas organizações tentam reduzir esses riscos e fragilidades do formato dos hackathons com a atuação com parceiros, principalmente em etapas de incubação das soluções vencedoras do concurso. Ao aproximar atores de instituições de ensino, pesquisa e 
desenvolvimento e setor privado, pode-se criar um ambiente de maior fluxo de conhecimento, contribuindo para o desenvolvimento das soluções (CHESBROUGH et al., 2006).

Outras áreas nos órgãos públicos foram identificadas pelos entrevistados, chamadas de Laboratórios Hackers, Laboratórios de Inovação ou Living Labs. Os laboratórios podem ser espaços para experimentar novas soluções, políticas e ferramentas de governança no setor público, além da colaboração para a resolução de problemas (GASCÓ, 2016; YAZDIZADEH; TAVASOLI, 2016). Nos casos aqui relatados, visam mediar a relação entre usuários e organizações do setor público e privado para capturar insights para serviços públicos. Esses laboratórios atuam no suporte às empresas para criar um ecossistema de inovação que beneficie tanto o setor público, quanto o privado, enfatizando a participação dos cidadãos.

A maior parte dos aplicativos morre. O grupo não tem interesse em manter. E pode ser que o aplicativo seja incubado por outras iniciativas. Alguns projetos podem ser mantidos por faculdades. Alguns aplicativos foram incorporados ao Laboratório Hacker. Incluíram funcionalidades e melhoraram visual. (Entrevista 7)

Com base nos resultados expostos, é possível perceber que os hackathons são uma importante estratégia de citizen-sourcing com vistas à inovação aberta. Entretanto, seu formato possui fragilidades com relação aos custos envolvidos em sua organização e à sustentabilidade das soluções criadas. Algumas organizações adaptaram o formato e trouxeram outros atores e áreas para aumentar as chances de sucesso das soluções criadas, como o caso do Laboratório Hacker.

A partir disso, é proposto um framework de promoção de hackathons baseado nos resultados desta pesquisa (Quadro 2):

Quadro 2 - Framework de promoção de hackathons no setor público, baseado em Linders (2012) e Mergel (2015)

\begin{tabular}{|l|l|}
\hline \multicolumn{1}{|c|}{ Fases } & \multicolumn{1}{c|}{ Etapas e aspectos críticos } \\
\hline Planejamento & $\begin{array}{l}\text { - Definição de objetivos: geração de inovações, participação social; aumento } \\
\text { da transparência; fortalecimento do ambiente/rede de inovação; aceleração de } \\
\text { mudanças organizacionais; aproximação de atores para inovação (cidadãos, } \\
\text { setor público, setor privado e instituções de ensino e pesquisa); aprendizagem; } \\
\text { melhoria da qualidade das decisões; melhoria da imagem institucional; } \\
\text { melhoria da consciência de problemas sociais; redução de custos; formulação } \\
\text { de políticas. } \\
\text { - Definição do formato: período de realização da iniciativa, regulamentos } \\
\text { (edital, concursos, entre outros) e divulgação. }\end{array}$ \\
\hline Construção & $\begin{array}{l}\text { - Geração de ideias e desenvolvimento de protótipos: formação de equipes e } \\
\text { participação social no levantamento de ideias e criação dos protótipos de } \\
\text { soluções. } \\
\text { - Avaliação dos protótipos: comissão avaliadora; sugestões de melhorias aos } \\
\text { protótipos das soluções considerando aspectos de legalidade, viabilidade e } \\
\text { sustentabilidade; premiação (recompensas financeiras e não financeiras). }\end{array}$ \\
\hline
\end{tabular}


(CONTINUA)

\begin{tabular}{|l|l|}
\hline \multirow{2}{*}{ Implantação } & $\begin{array}{l}\text {-Incubação: via setor privado (incubadoras e financiadores); via setor público } \\
\text { (concursos, licitações ou departamentos de apoio especializados como } \\
\text { laboratórios hackers); via instituições de ensino, pesquisa e desenvolvimento } \\
\text { (incubadoras). }\end{array}$ \\
\hline Avaliação e monitoramento & $\begin{array}{l}\text { - Monitoramento da continuidade das soluções } \\
\text { - Avaliação dos resultados dos hackathons e soluções desenvolvidas }\end{array}$ \\
\hline
\end{tabular}

Fonte: elaboração própria.

Os hackathons possuem três fases. Inicialmente é realizado o planejamento da iniciativa. Em seguida a construção das soluções e, por fim, a implantação das mesmas. Essas etapas são próximas às elaboradas no modelo de quatro fases da inovação aberta no setor público de Mergel (2015), apresentado na Seção de Referencial Teórico. Entretanto, a partir dos relatos dos entrevistados, é possível perceber que a sustentabilidade e avaliação dos resultados das soluções criadas pelos cidadãos são aspectos muito importantes, mas não abordados nas quatro fases do modelo de Mergel (2015). Nesse sentido, é importante uma etapa de avaliação e monitoramento das soluções construídas em parceria com os diversos atores.

Devido às limitações relacionadas à sustentabilidade das soluções, torna-se necessário definir mecanismos de implantação das soluções. Entre os casos analisados, a parceria com outros atores como o setor privado e as instituições de ensino, pesquisa e desenvolvimento pode apoiar a implantação das soluções por meio de incubação. O próprio setor público também pode utilizar outras formas de apoio à implantação das soluções, como a contratação via concursos ou licitações ou até com estruturas organizacionais mais receptivas à interação com o cidadão, como o caso dos Laboratórios Hackers e Laboratórios de Inovação.

\section{CONSIDERAÇÕES FINAIS}

Esta pesquisa teve como objetivo investigar a finalidade e resultados das iniciativas de hackathons utilizadas como estratégia de citizen-sourcing para inovação aberta no setor público brasileiro, considerando a perspectiva de agentes públicos promotores das iniciativas. $\mathrm{O}$ levantamento dos hackathons promovidos por organizações do setor público brasileiro mostrou que a iniciativa está em crescimento e vem sendo aplicada com diferentes objetivos. Os resultados da pesquisa podem ser resumidos nos seguintes pontos:

a) O primeiro hackathon realizado no setor público brasileiro ocorreu em 2012 e foi promovido pela Câmara Municipal de São Paulo. A partir de 2012, observou-se o crescimento no número de hackathons no setor público, tendo sido contabilizado um total de 47 iniciativas até novembro de 2016. Essas iniciativas se concentram, principalmente, 
no nível municipal e federal, em São Paulo, no Paraná e no Distrito Federal. A maioria das iniciativas $(73 \%)$ dura entre dois e três dias, ocorrendo normalmente no fim de semana e contando com recompensas financeiras (prêmios de até $\mathrm{R}$ \$30.000,00) e não financeiras (viagens, "miniestágios", cursos, programas de mentoria e incubação, dentre outros);

b) Os principais propósitos de organizações do setor público brasileiro na promoção de hackathons sob a ótica dos agentes públicos entrevistados foram: geração de inovações, promoção da participação social, aumento da transparência, fortalecimento do ambiente e da rede de inovação, aproximação de atores para a inovação (setor privado, setor público e instituições de ensino, pesquisa e desenvolvimento) e aceleração de mudanças organizacionais internas;

c) Para a maioria dos agentes públicos entrevistados, os objetivos dos hackathons têm sido alcançados, e estes merecem ser realizados novamente. Entre os principais resultados, destacam-se: aumento do número de novos negócios na região, maior apropriação e abertura de dados da organização do setor público e mudanças na percepção de funcionários da organização. Entretanto, ressaltam-se como argumentos para não voltar a realizar novos hackathons: a qualidade das soluções desenvolvidas aquém da expectativa e a sustentabilidade das soluções (grande parte das soluções é abandonada após a realização do hackathon);

d) Propôs-se um framework de promoção de hackathons com a finalidade de potencializar o alcance dos objetivos das iniciativas. Este framework é composto por quatro fases (planejamento, construção, implantação e, por fim, avaliação e monitoramento) e diversas etapas e aspectos críticos a serem considerados na organização dos eventos. Em especial, destaca-se a aproximação de organizações do setor público com outras organizações (públicas, privadas e do terceiro setor) para atuação mais próxima com hackers na incubação, monitoramento da continuidade das soluções e avaliação dos impactos das soluções desenvolvidas.

Este trabalho contribui com o aumento do conhecimento sobre as iniciativas de hackathons, citizen-sourcing e inovação aberta no setor público brasileiro. Devido à característica exploratório-descritiva da pesquisa, os resultados apresentados abrem espaço para estudos futuros mais profundos sobre o fenômeno, principalmente sobre outras variáveis que influenciam a participação e o engajamento de cidadãos em hackathons no setor público. Apesar de serem adotados como estratégia de citizen-sourcing, os hackathons diferem significativamente de outras estratégias, tanto em formato, nível de envolvimento e interação entre cidadãos e agentes públicos. 
Também se destaca a necessidade de mais pesquisas ligadas à qualidade das soluções. A sustentabilidade das soluções foi ponto de destaque nas entrevistas com agentes públicos. Presume-se que cidadãos possam sentir-se frustrados após o esforço em criar soluções para problemas públicos quando estas não são implementadas. Assim, levantam-se questionamentos sobre iniciativas de citizen-sourcing, principalmente para aquelas envolvendo ideias dos cidadãos não implementadas: a) os protótipos e ideias apresentadas pelos cidadãos são implementadas pelo setor público ou outras organizações?; b) as organizações do setor público possuem instrumentos e capacidades internas para absorver as soluções e ideias criadas pelos cidadãos em estratégias de citizen-sourcing?; c) quais são as consequências da não implementação das ideias sugeridas pelos cidadãos em sua predisposição em participar novamente de iniciativas de citizen-sourcing?; e d) quais os impactos da não implementação das ideias sugeridas pelos cidadãos na imagem das organizações promotoras da iniciativa e no setor público em geral?

Por fim, esta pesquisa trouxe insumos à tomada de decisão dos agentes públicos ao propor um framework de promoção de hackathons. Ao aprimorar as experiências de citizensourcing, o cidadão poderá notar que o setor público, quando adota tais iniciativas, não tem somente o objetivo de seguir um modismo ou parecer "cool" (NAM, 2012), mas demanda melhorias e soluções reais de que o país necessita.

\section{REFERÊNCIAS}

ALVES-MAZZOTTI, Alda Judith. Usos e abusos dos estudos de caso. Cadernos de pesquisa, v. 36, n. 129, p. 637-651, 2006. Disponível em: <http://www.scielo.br/pdf/cp/v36n129/a0736129>. Acesso em: 11 out. 2015.

ANGELIDIS, Pantelis et al. The hackathon model to spur innovation around global mHealth. Journal of Medical Engineering \& Technology, v. 40, n. 7-8, p. 392-399, 2016.

BARDIN, L. Analise de Conteúdo. Edição Por. Lisboa, Portugal: EDIÇÕES 70, LDA, 1977.

BOMMERT, Ben. Collaborative Innovation in the Public Sector. International public management review, v. 11, n. 1, p. 15-33, 2010.

BRABHAM, Daren. Crowdsourcing. [s. 1.]: MIT Press. BRISCOE, Gerard, 2013. 
BRISCOE, Gerard Briscoe; MULLIGAN, Catherine. Digital Innovation: The Hackathon Phenomenon. Creativeworks London, n. 6, p. 1-13, 2014. (Creativeworks London). Disponível em: <http://www.creativeworkslondon.org. uk/wpcontent/uploads/2013/11/Digital-Innovation-The-Hackathon-Phenomenon1.pdf >. Acesso em: 11 out. 2015.

CÂMARA DOS DEPUTADOS. Laboratório Hacker da Câmara coordena hackathon na Conferência Mundial sobre o Parlamento Eletrônico 2016. Disponível em: <https://www2.camara.leg.br/camaranoticias/radio/materias/RADIOAGENCIA/512186LABORATORIO-HACKER-DA-CAMARA-COORDENA-HACKATHON-NACONFERENCIA-MUNDIAL-SOBRE-O-PARLAMENTO-ELETRONICO-2016.html>. Acesso em 11/07/2016.

CHESBROUGH, H. The era of open innovation MIT Sloan Management Review. Spring, p. 35-36, 2003.

; VANHAVERBEKE, W.; WEST, J. Open innovation: Researching a new paradigm. First. Oxford: Oxford University Press, 2006.

COYNE, Imelda T. Sampling in qualitative research. Purposeful and theoretical sampling; merging or clear boundaries?. Journal of advanced nursing, v. 26, n. 3, p. 623-630, 1997.

CRESWELL, J W. Research Design: Qualitative, Quantitative, and Mixed Methods Approaches. [s. 1.]: SAGE Publications, 2014.

FARMER, Tracy et al. Developing and Implementing a Triangulation Protocol for Qualitative Health Research. Qualitative Health Research, v. 16, p. 377, 2006.

FERREIRA, Gabriel et al. Citizen-sourcing e inovação aberta no setor público: um panorama da publicação atual e perspectivas futuras. Em: XL Encontro da ANPAD. [s. n.], 2016, p. 119.

FERREIRA, Gabriel De Deus; FARIAS, Josivania Silva. The Motivation to Participate in Citizen-Sourcing and Hackathons in the Public Sector. Brazilian Administration Review, v. 15, n. 3, p. 1-22, 2018.

FREITAS, Rony Klay Viana de; DACORSO, Antonio Luiz Rocha. Inovação aberta na gestão pública: análise do plano de ação brasileiro para a Open Government Partnership. Rev. Adm. Pública, Rio de Janeiro, v. 48, n. 4, p. 869-888, 2014. 
GASCÓ, Mila. Living labs: Implementing open innovation in the public sector. Government Information Quarterly, 2016.

GAVENTA, John; BARRETT, Gregory. So What Difference Does it Make? Mapping the Outcomes of Citizen Engagement. Development, v. 2010, n. October, p. 1-72, 2010.

GODOY, A. S. Pesquisa qualitativa e suas possibilidades. Revista de Administração de Empresas, v. 35, n. pesquisa qualitativa, p. 57-63, 1995.

GUION, LA; DIEHL, DC; MCDONALD, D. Triangulation: Establishing the validity of qualitative studies. FCS6014, p. 1-3, 2002.

HILGERS, Dennis; IHL, Christoph. Citizensourcing: Applying the Concept of Open Innovation to the Public Sector. The International Journal of Public Participation, v. 4, n. $1,2010$.

HULGARD, Lars; FERRARINI, Adriane. Inovação social: rumo a uma mudança experimental na política pública? Ciências Sociais Unisinos, v. 46, n. 3, p. 256-263, 2010.

HYNES, Lisa et al. Strength In Numbers Hackathon: Using a novel technology-focused brainstorming activity to engage stakeholders in intervention development. European Health Psychologist, v. 18, n. 6, p. 287-293, 2016.

KARLSSON, Fredrik et al. Exploring user participation approaches in public e-service development. Government Information Quarterly, v. 29, n. 2, p. 158-168, 2012.

LINDERS, Dennis. From e-government to we-government: Defining a typology for citizen coproduction in the age of social media. Government Information Quarterly, 2012.

LUKENSMEYER, C. J.; TORRES, L. H. Citizen Sourcing: Citizen Participation in a Networked Nation. In: YANG, K.; BERGRUD, E. (Orgs.). Civic Engagement in a Network Society. Charlotte, North Carolina: Information Age Publishing, 2008, p. 207-233.

MARSHALL, Martin N. Sampling for qualitative research. Family practice, v. 13, n. 6, p. 522526, 1996. 
MAZZOTTI, AJ. Usos e abusos dos estudos de caso. Cadernos de pesquisa, v. 36, n. 129, p. 637-651, 2006. Disponível em: <http://www.scielo.br/pdf/cp/v36n129/a0736129>. Acesso em: 11 out. 2015.

MERGEL, I; DESOUZA, Kc. Implementing Open Innovation in the Public Sector: The Case of Challenge.gov. Public Administration Review, v. 73, n. 6, p. 882-890, 2013.

MERGEL, Ines. Opening Government: Designing Open Innovation Processes to Collaborate With External Problem Solvers. Social Science Computer Review, v. 33, n. 5, p. 599-612, 2015.

NAM, Taewoo. Suggesting frameworks of citizen-sourcing via Government 2.0. Government Information Quarterly, v. 29, n. 1, p. 12-20, 2012.

OECD. Citizens as Partners: Information, Consultation and Public Participation in PolicyMaking. OECD Handbook, v. 20, n. 424473, p. 163-178, 2001.

OSBORNE, Stephen P. (Ed.). The new public governance: Emerging perspectives on the theory and practice of public governance. USA and CANADA, Routledge, 2010.

PATTON, M. Qualitative evaluation and research methods. Beverly Hills, CA: Sage, 1990.

POWELL, Maria C; COLIN, Mathilde. Meaningful Citizen Engagement in Science and Technology What Would it Really Take? Science Communication, v. 30, n. 1, p. 126-136, 2008.

REDDEL, Tim; WOOLCOCK, Geoff. From consultation to participatory governance? A critical review of citizen engagement strategies in Queensland. Australian Journal of Public Administration, v. 63, n. 3, p. 75-87, 2004.

THAPA, Basanta E. P. et al. Citizen involvement in public sector innovation: Government and citizen perspectives. Information Polity, v. 20, n. 1, p. 3-17, 2015.

YANG, Kaifeng. Public Administrators' trust in citizens: a missing link in citizen involvement efforts. Public Administration Review, v. 65, n. 3, p. 273-285, 2005.

YAZDIZADEH, Ali; TAVASOLI, Ali. Living Labs as a Tool for Open Innovation: a Systematic Review. International Journal of Humanities and Cultural Studies ISSN, p. 2356-5926, 2016. 